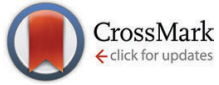

Cite this: Phys. Chem. Chem. Phys., 2014, 16, 24672

Received 22nd August 2014 Accepted 3rd October 2014

DOI: $10.1039 / c 4 c p 03765 k$

www.rsc.org/pccp

\section{Spin mixing at level anti-crossings in the rotating frame makes high-field SABRE feasible $\dagger$}

\author{
Andrey N. Pravdivtsev, ${ }^{a b}$ Alexandra V. Yurkovskaya, ${ }^{a b}$ Hans-Martin Vieth ${ }^{c}$ and \\ Konstantin L. Ivanov*ab
}

A new technique is proposed to carry out Signal Amplification By Reversible Exchange (SABRE) experiments at high magnetic fields. SABRE is a method, which utilizes spin order transfer from para-hydrogen to the spins of a substrate in transient complexes using suitable catalysts. Such a transfer of spin order is efficient at low magnetic fields, notably, in the Level Anti-Crossing (LAC) regions. Here it is demonstrated that LAC conditions can also be fulfilled at high fields in the rotating reference frame under the action of an RF-field. Spin mixing at LACs allows one to polarize substrates at high fields as well; the achievable NMR enhancements are around 360 for the ortho-protons of partially deuterated pyridine used as a substrate and around 700 for $\mathrm{H}_{2}$ and substrate in the active complex with the catalyst. High-field SABRE effects have also been found for several other molecules containing a nitrogen atom in the aromatic ring.

Signal Amplification By Reversible Exchange (SABRE) is a technique suggested in $2009^{1}$ that has drawn considerable attention during the last few years. ${ }^{2-9}$ The SABRE method, which is a variant of the ParaHydrogen Induced Polarization (PHIP) technique, ${ }^{10}$ is based on transfer of spin order from parahydrogen, which is the $\mathrm{H}_{2}$ molecule in its singlet spin state, to a substrate in a transient complex using a suitable catalyst. As a result, the substrate gets hyperpolarized and its Nuclear Magnetic Resonance (NMR) signals are strongly enhanced. By using this method, molecules such as pyridine, ${ }^{1}$ derivatives of pyridine and pyrazole, ${ }^{5}$ as well as purine nucleotides ${ }^{9}$ can be hyperpolarized. Since the SABRE technique does not require chemical modification of molecules, in contrast to the classical PHIP, which requires binding of $\mathrm{H}_{2}$ to a substrate, it is potentially a more general method, which is promising for many new NMR

\footnotetext{
${ }^{a}$ International Tomography Center, Siberian Branch of the Russian Academy of Science, Institutskaya 3a, Novosibirsk, 630090, Russia.

E-mail: ivanov@tomo.nsc.ru; Fax: +7(383)333-1399; Tel: +7(383)330-8868

${ }^{b}$ Novosibirsk State University, Pirogova 2, Novosibirsk, 630090, Russia

${ }^{c}$ Institut für Experimentalphysik, Freie Universität Berlin, Arnimallee 14, Berlin, 14195, Germany

$\dagger$ Electronic supplementary information (ESI) available: Details of sample preparation; calculation algorithm; results for Crabtree's catalyst and other substrates; NMR parameters of the spin systems. See DOI: 10.1039/c4cp03765k
}

applications. Because of the cyclic character of the process SABRE makes continuous production of hyperpolarization ${ }^{9}$ possible and $^{-1}$ enables measurements with nanomolar concentrations of substrate. ${ }^{7}$ Signal enhancement provided by the SABRE method is often 2-3 orders of magnitude as compared to thermal NMR signals at high magnetic fields. As there is high demand for signal enhancement in NMR the SABRE method is extremely promising for utilization in NMR spectroscopy and imaging.

One of the problems with the SABRE technique is that it is most efficient at low fields, whereas in NMR spectroscopy it is advantageous to run measurements at high fields where the spectral resolution is much better. Thus, to perform high-resolution NMR studies with SABRE-derived hyperpolarization one needs to perform field-cycling, which can be technically difficult, and also imposes limitations on the hyperpolarization lifetime. For practical purposes it is advantageous to polarize nuclear spins by SABRE directly at the high field of an NMR spectrometer or imager. In a recent paper Barskiy et al. ${ }^{8}$ have shown that SABRE is manifest at high fields and signal enhancements of about 5 are achievable. In this case polarization of substrates is presumably due to cross-relaxation in the complex; the resulting spin polarization is orders of magnitude lower than at low fields, where spin order transfer is an efficient coherent process.

Here we present an approach, which makes efficient high-field SABRE feasible. The transfer of spin order is fast and provides NMR signal enhancements of up to 360 for free substrates and around 700 for protons of $\mathrm{H}_{2}$ and of the substrate molecules bound to active catalysts. As the key idea we use the fact that polarization transfer, in general, ${ }^{11-16}$ and SABRE, in particular, ${ }^{17}$ is most efficient at nuclear spin Level Anti-Crossings (LACs) in the transient complex of parahydrogen, catalyst and substrate. Typically, such LACs occur ${ }^{17,18}$ at magnetic fields below 20-30 mT; nonetheless, the same ideas can be exploited when the spin system is brought to LACs in the rotating frame under the action of a strong RF-field (spin-locking field). We will explain the physical principles behind this technique and present experimental results for high-field SABRE under spinlocking conditions, RF-SABRE. The advantages and potential applications of the method are also discussed. 

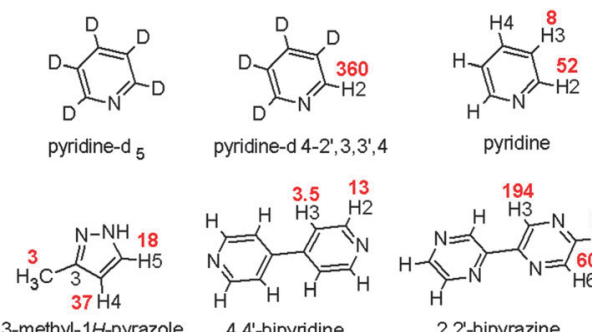

pyridine-d 4-2', 3,3', 4

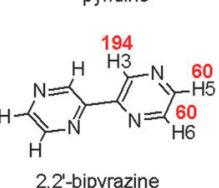

Chart 1 Chemical structures of substrates used; assignments of the proton positions and absolute values of the achieved NMR signal enhancements are also specified.

SABRE-derived polarization was produced in complexes of several substrates, see Chart 1 , with the parahydrogen at two different catalysts: Crabtree's catalyst ${ }^{19}$ and the recently proposed IrIMes catalyst. ${ }^{4}$ As an example the structure of the active complex of IrIMes with partially deuterated py with a single ortho-proton, as well as the reaction pathway leading to the SABRE effect are shown in Scheme 1. Here, for the sake of simplicity, we will mostly discuss SABRE effects for deuterated pyridine (py) with the abundance of protons of only $0.5 \%$ so that the substrate contains only one proton or no protons at all. Further experiments with protonated py and other substrates are presented in the ESI; $\dagger$ in these systems the spin dynamics is more complex and NMR signal enhancements are lower. SABRE experiments were carried out at the high field of a $200 \mathrm{MHz}$ spectrometer (magnetic field $B_{0}=4.7$ Tesla) in the following way: first we let the system relax to its thermal equilibrium at $B=B_{0}$. After that the $\mathrm{H}_{2}$ gas enriched in its para-component (92\% parahydrogen) is bubbled through the sample for $6 \mathrm{~s}$. During this period the dihydrogen gas is efficiently dissolved in the sample. After waiting for $1.5 \mathrm{~s}$, which is necessary to remove bubbles from the solution, an RF-field with amplitude $\nu_{1}$ and frequency $\nu_{\mathrm{rf}}$ (spin-locking) is turned on for $\tau_{\mathrm{rf}}$, which is varied in the range of 0.1-7 $\mathrm{s}$. The duration of the spin-locking period is long enough so that complex formation and dissociation can frequently occur; $\tau_{\mathrm{rf}}$ is smaller than the relaxation time of the protons of free py and comparable to the relaxation time of the py protons in the active complex. We checked that the polarization
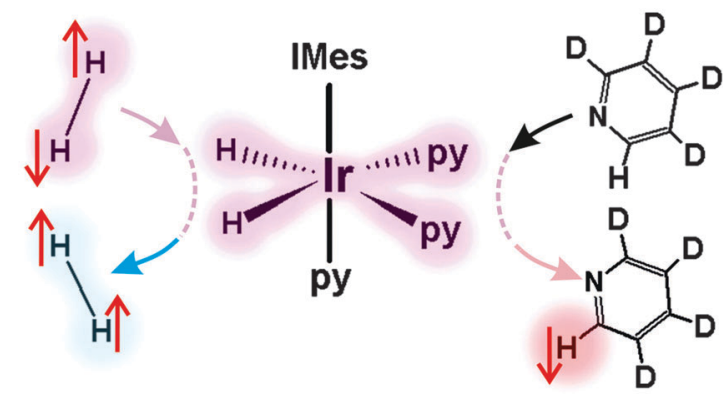

Scheme 1 SABRE formation in the complex of the IrIMes catalyst with parahydrogen and partially deuterated pyridine (py) having only one proton in the ortho-position, IMes = 1,3-bis(2,4,6-trimethylphenyl) imidazole2 -ylidene. Transfer of the initial singlet spin order (represented by antiparallel arrows) of the parahydrogen molecule entering the complex results in polarization of py (represented by "down" arrow); at the same time, the $\mathrm{H}_{2}$ molecule goes to its ortho-state (shown by "up" arrows). level gets saturated for py at $\tau_{\mathrm{rf}} \approx 5 \mathrm{~s}$ (while for the relaxation-based mechanism the saturation occurs after $100 \mathrm{~s}$ of bubbling $\left.{ }^{8}\right)$. When the parameters $\nu_{1}$ and $\nu_{\mathrm{rf}}$ are set properly (see below), the substrate acquires hyperpolarization. Transverse spin magnetization (free induction) is seen immediately after switching off the RF-field. By taking the Fourier transform of the free induction decay we obtained NMR spectra with enhanced lines. If instead of transverse magnetization longitudinal magnetization is desired it is simple to generate it by applying a $\pi / 2$ pulse 90 degrees out-of-phase with respect to the spin-locking RF-field.

Typical RF-SABRE spectra are shown in Fig. 1 together with the thermal NMR spectrum recorded before bubbling the sample with parahydrogen. In the RF-SABRE spectra the phase of the lines depends on the frequency $\nu_{\mathrm{rf}}$. The enhancements achieved for the substrate are about 360, which is much higher than the reported high-field SABRE effect ${ }^{8}$ based on 'spontaneous' (i.e. without RF-excitation) polarization transfer to the substrate. Polarization is produced predominantly for the ortho-protons of py; however, a small amount of polarization is also seen for other protons. In addition, we observed polarized signals of $\mathrm{H}_{2}$ in the solvent bulk $\left(\mathrm{H}_{2}\right)$ and at the catalyst $\left(\mathrm{c}-\mathrm{H}_{2}\right)$ with the opposite sign to those of the ortho-protons of py. The signal enhancement for the two $\mathrm{H}_{2}$ signals is different for different substrates; the highest enhancement achieved when 2,2'-bipyrazine was used as a substrate was about 100-200 for $\mathrm{H}_{2}$ and about 700 for $\mathrm{c}-\mathrm{H}_{2}$ (see ESI $\dagger$ ).

Let us now give the explanation of the SABRE mechanism and also discuss how the enhancement depends on the parameters of the RF-field. Here we will not go into full details of the spin dynamics, but provide a descriptive and reasonably simple treatment of the problem under study. Let us write down the Hamiltonian of the spin system under RF-excitation. For simplicity, we assume here that the substrate has only one spin (M-spin) and that the chemical shifts of the protons coming from parahydrogen ( $\mathrm{AA}^{\prime}$-protons) have identical chemical shifts in the complex. This is the minimal spin system, in which the SABRE effect can be formed; it also

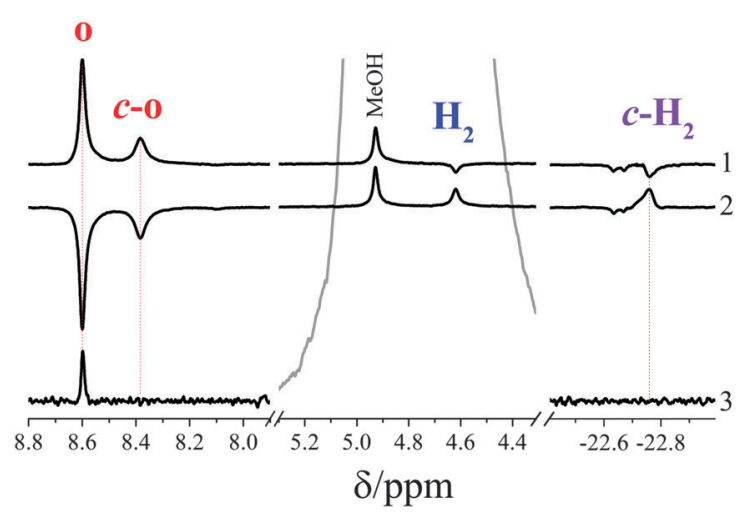

Fig. $1{ }^{1} \mathrm{H}$ RF-SABRE spectra obtained using the IrIMes catalyst after applying an RF-field with an amplitude of $28.6 \mathrm{kHz}$ and frequency positions $\nu_{\mathrm{rf}}=$ $-7.0 \mathrm{ppm}$ (trace 1), $-7.35 \mathrm{ppm}$ (trace 2). Deuterated py (proton abundance of $0.5 \%$ ) was used as a substrate; positions of different protons are assigned. Here $\mathrm{O}$ and $\mathrm{c}-\mathrm{O}$ denote the ortho-protons of py in the bulk and are bound to the catalyst; $\mathrm{H}_{2}$ and $\mathrm{c}-\mathrm{H}_{2}$ denote dihydrogen in the bulk and in the catalyst, respectively. The thermal NMR spectrum obtained after 128 acquisitions with a $\pi / 2$-read pulse is also shown for comparison (trace 3 ). 
corresponds to the case of deuterated py having only one proton or only residual protons. Then the Hamiltonian in the rotating frame is:

$$
\begin{aligned}
\hat{H}_{\mathrm{rf}}= & -\delta \nu_{\mathrm{A}}\left(\hat{I}_{\mathrm{A} z}+\hat{I}_{\mathrm{A}^{\prime} z}\right)-\delta \nu_{\mathrm{M}} \hat{I}_{\mathrm{M} z}+\hat{J}_{\mathrm{AA^{ \prime }}}\left(\hat{\mathbf{I}}_{\mathrm{A}^{\prime}} \hat{\mathbf{I}}_{\mathrm{A}^{\prime}}\right)+J_{\mathrm{AM}}\left(\hat{\mathbf{I}}_{\cdot} \cdot \hat{\mathbf{I}}_{\mathrm{M}}\right) \\
& +J_{\mathrm{A}^{\prime} \mathbf{M}}\left(\hat{\mathbf{I}}_{\mathrm{A}^{\prime}} \cdot \hat{\mathbf{I}}_{\mathrm{M}}\right)-\nu_{1}\left(\hat{I}_{\mathrm{Ax}}+\hat{I}_{\mathrm{A}^{\prime} x}+\hat{I}_{\mathrm{M} x}\right)
\end{aligned}
$$

where $\delta \nu_{i}$ are the NMR frequencies of spins in the rotating frame; $J_{i j}$ are the scalar spin-spin interaction constants. Here it is more suitable to take the following terms

$$
\hat{V}=J_{\mathrm{AM}}\left(\hat{\mathbf{I}}_{\mathrm{A}} \cdot \hat{\mathbf{I}}_{\mathrm{M}}\right)+J_{\mathrm{A}^{\prime} \mathrm{M}}\left(\hat{\mathbf{I}}_{\mathrm{A}^{\prime}} \cdot \hat{\mathbf{I}}_{\mathrm{M}}\right)
$$

as a small perturbation into consideration. This is a good approximation when $J_{\mathrm{AA}^{\prime}}$ is by far the largest coupling in the spin system. The main part of the Hamiltonian, $\hat{H}_{0}=\hat{H}_{\mathrm{rf}}-\hat{V}$, can be most conveniently written in the so-called Doubly Tilted Frame (DTF) where the spins in each group (AA'-protons and $\mathrm{M}$-proton) are quantized along their effective fields in the rotating frame, see Fig. $2 \mathrm{a}$. In this frame $\hat{H}_{0}$ takes the form:

$$
\hat{H}_{0, \mathrm{dtf}}=-\nu_{\mathrm{A}, \mathrm{dtf}}\left(\hat{I}_{\mathrm{A} z}+\hat{I}_{\mathrm{A}^{\prime} z}\right)-\nu_{\mathrm{M}, \mathrm{dtf}} \hat{I}_{\mathrm{Mz}}+\hat{J}_{\mathrm{AA}^{\prime}}\left(\hat{\mathbf{I}}_{\mathrm{A}} \cdot \hat{\mathbf{I}}_{\mathrm{A}^{\prime}}\right)
$$

where $\nu_{\mathrm{A}, \mathrm{dtf}}=\sqrt{\left(\delta \nu_{\mathrm{A}}\right)^{2}+\nu_{1}^{2}}$ and $\nu_{\mathrm{M}, \mathrm{dtf}}=\sqrt{\left(\delta \nu_{\mathrm{M}}\right)^{2}+\nu_{1}^{2}}$. In this frame there are two level crossings occurring when ${ }^{17}$

$$
\nu_{\mathrm{A}, \mathrm{dtf}}-\nu_{\mathrm{M}, \mathrm{dtf}}= \pm J_{\mathrm{AA}^{\prime}}
$$

These crossings are between the energy levels corresponding to the state composed of the singlet configuration of the $\mathrm{AA}^{\prime}$-protons and $\beta$ of the $\mathrm{M}$-proton, $|\mathrm{S} \beta\rangle$, and of the lower triplet state of the $\mathrm{AA}^{\prime}$-protons and $\alpha$ of the M-proton, $\left|\mathrm{T}_{-} \alpha\right\rangle$, in the DTF (corresponding to the "-_" sign in eqn (4)) or between $|\mathrm{S} \alpha\rangle$ and the upper triplet state of the $\mathrm{AA}^{\prime}$-protons and $\beta$ of the M-proton, $\left|T_{+} \beta\right\rangle$ (corresponding to the "+" sign in eqn (4)); initially only the levels with singlet character of the $\mathrm{AA}^{\prime}$-spins are populated. At the level crossing points the perturbation term $\hat{V}$ becomes important: when $J_{\mathrm{AM}} \neq J_{\mathrm{A}^{\prime} \mathrm{M}}$ it mixes the states and turns the crossing into an LAC. Consequently, the populations of the involved levels are mixed. When the
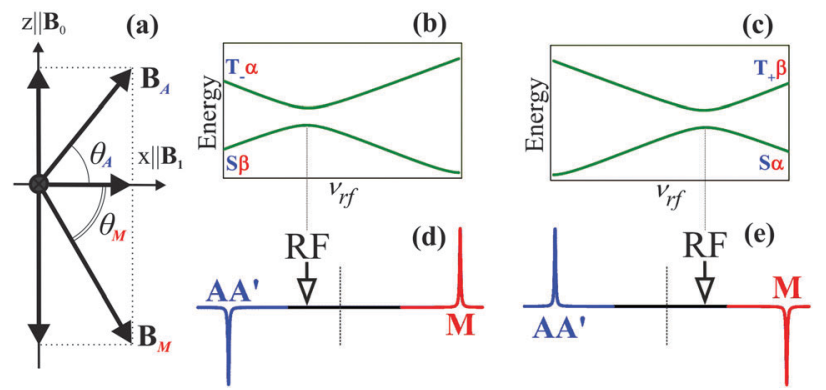

Fig. 2 Schematic representation of the axes of the doubly tilted frame (DTF) (a): spins are quantized along the new axes, $B_{\mathrm{A}}$ and $B_{M}$; they are inclined with respect to the $x$-axis, the inclination angles being $\theta_{A}$ and $\theta_{M}$. In (b) and (c) we show the LACs in the DTF between the levels $|S \beta\rangle$ and $\left|T_{-} \alpha\right\rangle$ and between the levels $|S \alpha\rangle$ and $\left|T_{+} \beta\right\rangle$. In (d) and (e) the NMR spectrum resulting from spin mixing at each LAC (with the position of the applied RF-field indicated by an arrow) is shown under the corresponding energy diagram. system is brought to the LAC between the $|\mathrm{S} \alpha\rangle$ and $\left|\mathrm{T}_{+} \beta\right\rangle$ states, see Fig. $2 \mathrm{~b}$, this kind of mixing results in positive polarization of the $\mathrm{AA}^{\prime}$-spins along their effective field in the DTF and negative polarization of the $\mathrm{M}$-spin along its effective field in the DTF. For the other LAC (Fig. 2c) the resulting polarization is of the same size, but opposite sign for both the $\mathrm{AA}^{\prime}$-protons and the M-proton. This simple model leads to two important consequences: (i) the RF-frequency dependence of polarization is anti-symmetric with respect to the center of the spectrum, $\left(\nu_{\mathrm{A}}+\nu_{\mathrm{M}}\right) / 2$; (ii) the polarization of the $\mathrm{AA}^{\prime}$-protons $\left(\mathrm{H}_{2}\right)$ has an opposite sign to that of the M-proton belonging to the substrate.

Finally, let us discuss the conditions for having an LAC in the DTF. From eqn (4) it follows that the two frequencies, $\nu_{\mathrm{A}, \mathrm{dtf}}$ and $\nu_{\mathrm{M} \text {,dtf }}$, should have a difference equal to $\pm J_{\mathrm{AA}^{\prime}}$, which is achieved when the frequency $\nu_{\mathrm{rf}}$ is placed almost at $\left(\nu_{\mathrm{A}}+\nu_{\mathbf{M}}\right) / 2$ having only a small offset from this value (either positive or negative). In addition, to make the mixing of the crossing levels efficient it is required ${ }^{20}$ that the angle between the two quantization axes of the DTF, $\Theta=\theta_{\mathrm{A}}+\theta_{\mathrm{M}}$, deviates from $\pi$, see Fig. $2 \mathrm{a}$. This condition is achieved when $\nu_{1}$ is sufficiently large, i.e., it is larger than or at least comparable to $\left(\nu_{\mathrm{A}}-\nu_{\mathrm{M}}\right)$. In this situation in the course of mixing at the LAC regions the spins acquire magnetization along the $x$-axis in the non-tilted rotating frame, which is detected in our experiments.

The spectra 1 and 2 shown in Fig. 1 have been taken when the LAC conditions are exactly fulfilled. The RF-frequency $\nu_{\mathrm{rf}}$ is chosen such that it is placed almost at the 'center of the spectrum', that is, at $\nu_{\mathrm{rf}}=\left(\nu_{\mathrm{cH}_{2}}+\nu_{\mathrm{co}}\right) / 2$ (here $\nu_{\mathrm{co}}$ stands for the NMR frequency of the ortho-protons of equatorial py in the active complex) but is slightly detuned from this value. Positive and negative offsets result in different sign of polarization of the ortho-protons and $\mathrm{c}-\mathrm{H}_{2}$ and $\mathrm{H}_{2}$, in full accordance with our theoretical treatment (compare curves 1 and 2). When the RFfrequency is such that the system stays away from an LAC the SABRE effect vanishes.

The full dependence of the high-field SABRE effect on the RF-field frequency is shown in Fig. 3 for the complex with the IrIMes catalyst. It is clearly seen that placing the frequency at the 'center of the spectrum' gives no polarization; however, there are positive and negative peaks in the $\nu_{\mathrm{rf}}$-dependence, at which the substrate is efficiently polarized, see Fig. 3a. The same shape of the frequency dependence is also found for $\mathrm{H}_{2}$ and $\mathrm{c}-\mathrm{H}_{2}$, but with the opposite phase, see Fig. $3 \mathrm{~b}$. The frequency dependences are in good agreement with the calculation. Details of the calculation method and the NMR parameters used in the calculation are also given in the ESI. $\dagger$ Analogous results are seen for RF-SABRE obtained using Crabtree's catalyst and also for RF-SABRE obtained using other substrates (see ESI $\dagger$ ).

RF-SABRE signal enhancements are summarized in Chart 1. It is seen that the effect is not limited solely to py with only one proton but is more general. Using the same physical principles it is possible to polarize other molecules containing nitrogen atoms in the aromatic ring. In fully protonated py the enhancement decreases but is still substantial; for fully protonated 2,2'-bipyrazine the enhancement is about 200. In other cases the NMR enhancement is larger than that given by the pure cross-relaxation mechanism. 


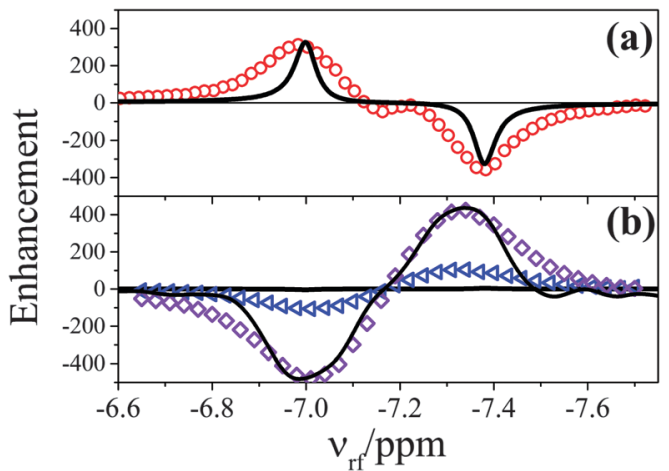

Fig. 3 Dependence of the RF-SABRE-induced NMR enhancement on $\nu_{\mathrm{rf}}$ for (a) the ortho-protons of free deuterated py (proton abundance is $0.5 \%$ ) and for (b) $\mathrm{H}_{2}$ (blue triangles) and $\mathrm{c}-\mathrm{H}_{2}$ (violet diamonds) when protonated py was used as a substrate. The RF-SABRE effect was observed for the IrIMes catalyst; lines show the simulation results.

Thus, despite the more complex spin dynamics in systems with several protons, it is feasible to achieve the RF-SABRE effect. Its dependence on the key experimental parameters, RF-field amplitude and frequency, is discussed in more detail in the ESI. $\dagger$

\section{Conclusions}

To summarize, we have proposed a method to perform RF-SABRE experiments at high magnetic fields. The method exploits mixing of spin states at LACs; the LAC conditions are fulfilled by turning on a strong RF-field with properly chosen amplitude and frequency. By varying the RF frequency one can also vary the phase of polarized NMR signals in a desirable way. These results can be well accounted for by the theoretical treatment described; also, a simple semi-qualitative explanation of our observations is given that is based on spin mixing in the doubly tilted frame of reference.

With the signal enhancement around 360, it is a significant step forward for establishing SABRE as a high-field technique. The method is relatively straightforward in use and only requires careful setting of the RF-field parameters. Since carrying out experiments at high magnetic fields is technically much easier than field-cycling and requires only standard NMR equipment we anticipate that our method will provide access to exciting applications in NMR spectroscopy and imaging providing a permanent source of nuclear hyperpolarization. Thus, the feasibility of high-field SABRE experiments opens new avenues in exploiting hyperpolarized spins.

\section{Acknowledgements}

This work was supported by the Russian Science Foundation (grant no. 14-13-01053). H.M.V. is thankful to the Alexander von Humboldt Foundation for financial support. We acknowledge Dr Pavel Petrov for providing us with the IrIMesCODCl pre-catalyst.

\section{Notes and references}

1 R. W. Adams, J. A. Aguilar, K. D. Atkinson, M. J. Cowley, P. I. Elliott, S. B. Duckett, G. G. Green, I. G. Khazal, J. LopezSerrano and D. C. Williamson, Science, 2009, 323, 1708-1711.

2 R. W. Adams, S. B. Duckett, R. A. Green, D. C. Williamson and G. G. R. Green, J. Chem. Phys., 2009, 131, 194505.

3 K. D. Atkinson, M. J. Cowley, P. I. P. Elliott, S. B. Duckett, G. G. R. Green, J. López-Serrano and A. C. Whitwood, J. Am. Chem. Soc., 2009, 131, 13362-13368.

4 M. J. Cowley, R. W. Adams, K. D. Atkinson, M. C. R. Cockett, S. B. Duckett, G. G. R. Green, J. A. B. Lohman, R. Kerssebaum, D. Kilgour and R. E. Mewis, J. Am. Chem. Soc., 2011, 133, 6134-6137.

5 E. B. Dücker, L. T. Kuhn, K. Münnemann and C. Griesinger, J. Magn. Reson., 2012, 214, 159-165.

6 M. Fekete, O. Bayfield, S. B. Duckett, S. Hart, R. E. Mewis, N. Pridmore, P. J. Rayner and A. Whitwood, Inorg. Chem., 2013, 52, 13453-13461.

7 N. Eshuis, N. Hermkens, B. J. A. van Weerdenburg, M. C. Feiters, F. P. J. T. Rutjes, S. S. Wijmenga and M. Tessari, J. Am. Chem. Soc., 2014, 136, 2695-2698.

8 D. A. Barskiy, K. V. Kovtunov, I. V. Koptyug, P. He, K. A. Groome, Q. A. Best, F. Shi, B. M. Goodson, R. V. Shchepin, A. M. Coffey, K. W. Waddell and E. Y. Chekmenev, J. Am. Chem. Soc., 2014, 136, 3322-3325.

9 J.-B. Hövener, N. Schwaderlapp, T. Lickert, S. B. Duckett, R. E. Mewis, L. A. R. Highton, S. M. Kenny, G. G. R. Green, D. Leibfritz, J. G. Korvink, J. Hennig and D. von Elverfeldt, Nat. Commun., 2013, 4, 2946.

10 J. Natterer and J. Bargon, Prog. Nucl. Magn. Reson. Spectrosc., 1997, 31, 293-315.

11 K. Miesel, K. L. Ivanov, A. V. Yurkovskaya and H.-M. Vieth, Chem. Phys. Lett., 2006, 425, 71-76.

12 A. S. Kiryutin, A. V. Yurkovskaya, R. Kaptein, H.-M. Vieth and K. L. Ivanov, J. Phys. Chem. Lett., 2013, 4, 2514-2519.

13 A. N. Pravdivtsev, A. V. Yurkovskaya, R. Kaptein, K. Miesel, H.-M. Vieth and K. L. Ivanov, Phys. Chem. Chem. Phys., 2013, 15, 14660-14669.

14 L. Buljubasich, M. B. Franzoni, H. W. Spiess and K. Münnemann, J. Magn. Reson., 2012, 219, 33.

15 M. B. Franzoni, D. Graafen, L. Buljubasich, L. M. Schreiber, H. W. Spiess and K. Münnemann, Phys. Chem. Chem. Phys., 2013, 15, 17233-17239.

16 K. L. Ivanov, A. N. Pravdivtsev, A. V. Yurkovskaya, H.-M. Vieth and R. Kaptein, Prog. Nucl. Magn. Reson. Spectrosc., 2014, 81, 1.

17 A. N. Pravdivtsev, A. V. Yurkovskaya, H.-M. Vieth, K. L. Ivanov and R. Kaptein, ChemPhysChem, 2013, 14, 3327-3331.

18 J.-B. Hövener, S. Knecht, N. Schwaderlapp, J. Hennig and D. von Elverfeldt, ChemPhysChem, 2014, 15, 2636.

19 R. H. Crabtree, M. Lavin and L. Bonneviot, J. Am. Chem. Soc., 1986, 108, 4032.

20 A. N. Pravdivtsev, A. V. Yurkovskaya, N. N. Lukzen, H.-M. Vieth and K. L. Ivanov, Phys. Chem. Chem. Phys., 2014, 16, 18707. 